\title{
Knowledge and Awareness About Plasma Substitutes Among Dental Students
}

\author{
Trishala A 1 , Dhanraj Ganapathy ${ }^{2}$ and M.P.Santhosh Kumar ${ }^{3}$ \\ ${ }^{1}$ Saveetha Dental College and Hospitals Saveetha Institute of Medical \\ and Technical Sciences Saveetha University Chennai, India \\ ${ }^{2}$ Head of department Department of Prosthodontics Saveetha Dental College and Hospitals \\ Saveetha Institute of Medical and Technical Sciences Saveetha University Chennai, India \\ ${ }^{3}$ Reader Department of Oral and Maxillofacial Surgery Saveetha Dental College and Hospitals \\ Saveetha Institute of Medical and Technical Sciences Saveetha University Chennai, India
}

\begin{abstract}
Plasma substitutes aim to replace a fluid which is colloidal in nature by virtue of its content of different species of highly specialized proteins. They include any liquid used to replace blood plasma, usually a saline solution, often with serum albumins, dextrans or other preparations. Considering the importance and uses of plasma substitutes, the aim of this questionnaire study was to assess the awareness about plasma substitutes among dental students in a university setting. A structured self assessed online questionnaire having 15 questions on plasma substitutes was prepared and distributed to the dental students who have clinical exposure including 3rd BDS, final BDS, interns and postgraduates of all specialities. It was circulated using an online software, survey planet and the response was collected through it. Statistical analysis was done using SPSS software. Considering the responses, it can be interpreted that the knowledge regarding plasma substitutes is above average among dental students. Also, postgraduate students were more knowledgeable than undergraduate students. Interns showed more awareness than third year or final year undergraduate students. Educational workshops, conferences and CDE's are essential for both undergraduates and postgraduates to improve their knowledge regarding various plasma substitutes and their adverse reactions
\end{abstract}

KEY WORDS: AWARENESS; DENTAL STUDENTS; DEXTRANS; PLASMA SUBSTITUTES.

\section{INTRODUCTION}

Blood and blood products have limited availability and there is increasing concern over infectious and immunologic risks and the costs involved in obtaining, storing, crossmatching, processing and dispersing blood

\section{ARTICLE INFORMATION}

*Corresponding Author: kaarthikeyan@saveetha.com

Received 15th June 2020 Accepted after revision 10th August 2020

Print ISSN: 0974-6455 Online ISSN: 2321-4007 CODEN: BBRCBA

Thomson Reuters ISI Web of Science Clarivate Analytics USA and Crossref Indexed Journal

\section{Clarivate
Analytics}

NAAS Journal Score 2020 (4.31) SJIF: 2020 (7.728)

A Society of Science and Nature Publication,

Bhopal India 2020. All rights reserved.

Online Contents Available at: http//www.bbrc.in/

Doi: http://dx.doi.org/10.21786/bbrc/13.7/57 and blood products continue to rise(Grobbelaar and Smart, 1967). There is, therefore, a clear role for effective blood and plasma substitutes(Stehling, Zauder and Rogers, 1975). Not only do these solutions provide a costeffective alternative to blood products, they also allow conservation of this limited resource for those patients who most need it(Stehling, Zauder and Rogers, 1975).

Plasma substitutes replace the fluid which is colloidal in nature by virtue of its content of different highly specialized proteins. This include liquid used to replace blood plasma, normally a saline solution, often with serum albumins, dextrans or other preparations(Underwood, Gowing and Johnston, 1967). These substances do not enhance the oxygen-carrying capacity of blood, but merely replace the volume(Kirch, Kühler and Gizycki,

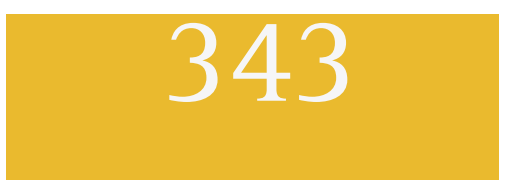


1979). They are also used to treat dehydration. They can be used to expand and maintain blood volume in shock arising from conditions such as burns or septicaemia. Plasma substitutes can be used as an immediary shortterm measure to treat massive haemorrhage until blood is available, but large volumes of some plasma substitutes can increase the risk of bleeding by depleting coagulation factors(Lamke and Liljedahl, 1976).

Following Starling's concepts in 1896 of colloid osmotic pressure as a regulator of the plasma volume, interest arose in developing colloid plasma expanders(Stacca et al., 1989). The first colloid plasma substitutes were tested around the turn of this century, when gum acacia was used as a volume expander. This substance is a carbohydrate exudate from Acacia trees and was used clinically during World War I. It proved to be highly antigenic, however anaphylaxis was common following its infusion(Solanke, Khwaja and Madojemu, 1971). Gelatin solutions derived from collagen were also developed in the early 1900s and these too were used clinically during World War I. The plasma expansion following gelatin infusion is short lived because of rapid urinary excretion. Further, gelatin infusions are antigenic and anaphylaxis may occur, particularly with repeated infusions(Chattri, 2012).

Modern plasma substitutes include dextrans, hydroxyethyl starch, per fluoro-chemical emulsions and stroma-free hemoglobin(Sayman and Garrott Allen, 1959). Each of these is a colloid fluid with the potential for efficient plasma volume expansion. Stroma-free hemoglobin solutions and per fluoro-chemical emulsions not only expand plasma volume but can transport additional oxygen as well(Sewta, 2009). Considering the importance and uses of plasma substitutes, this study evaluates the level of awareness among dental students.

Previously our department has published extensive research on various aspects of prosthetic dentistry ('Evaluation of Corrosive Behavior of Four Nickelchromium Alloys in Artificial Saliva by Cyclic Polarization Test:An in vitro Study', 2017; Ganapathy, Kannan and Venugopalan, 2017; Jain, 2017a, 2017b; Ranganathan, Ganapathy and Jain, 2017; Ariga et al., 2018; Gupta, Ariga and Deogade, 2018; Anbu et al., 2019; Ashok and Ganapathy, 2019; Duraisamy et al., 2019; Varghese, Ramesh and Veeraiyan, 2019), this vast research experience has inspired us to research about the awareness about plasma substitutes among dental students in a university setting.

\section{MATERIAL AND METHODS}

The present study is an online based survey conducted among dental students of a University. Ethical approval was obtained from the institution's ethical committee. The number of people involved in this study include the guide, reviewer and principle investigator. A structured self assessed online questionnaire having 15 questions on plasma substitutes was prepared with the aim to assess the awareness among 215 dental students. The questions were validated and reviewed closely. Sampling was done by convenient sampling. The questionnaires were distributed to the dental students who have clinical exposure including 3rd BDS, final BDS, interns and postgraduates of all specialities. It was circulated using an online link from the survey planet and the response was collected through it. Only the completed surveys were included for analysis. The collected results were entered in Microsoft excel and statistical analysis was done using SPSS software (IBM SPSS Statistics 20.0). Frequency distribution of each response among the dental students and Chi-square association using Pearson correlation with a level of significance set at $p<0.05$ was done to determine the association between year of study and the responses for each question.

\section{RESULTS AND DISCUSSION}

The study was conducted among 215 dental students. Out of $215,20.93 \%$ were third year undergraduate students, $12.56 \%$ were final year undergraduate students, 34.42\% were interns and 32.09\% were postgraduates [Figure 1]. About $54.42 \%$ of students answered that both plasma protein solution and albumin were natural plasma substitutes [Figure 2]. Out of the 54.42\%, 24.65\% were postgraduates, $14.88 \%$ were interns, $7.91 \%$ were third year students and 6.98\% were final year undergraduate students. There was a significant association $(p=0.003)$ between the year of study and the response for the question, 'Which of the following are natural colloids?' [Figure 3]. About 54.88\% of the students answered correctly that both dextran and hydroxyethyl starch were synthetic plasma substitutes [Figure 4]. Among them, $25.58 \%$ were postgraduates, $19.53 \%$ were interns, $4.19 \%$ were final year undergraduates and $5.58 \%$ were third year undergraduate students. There was a significant association $(p=0.000)$ between the year of study and the response for the question, 'Which of the following are synthetic colloids?' [Figure 5].

Figure 1: Pie chart represents the year of study of dental students included in this study. Majority of the study population were interns (34.42\%), followed by postgraduates (32.09\%), third year students(20.93\%) and final year students (12.56\%).

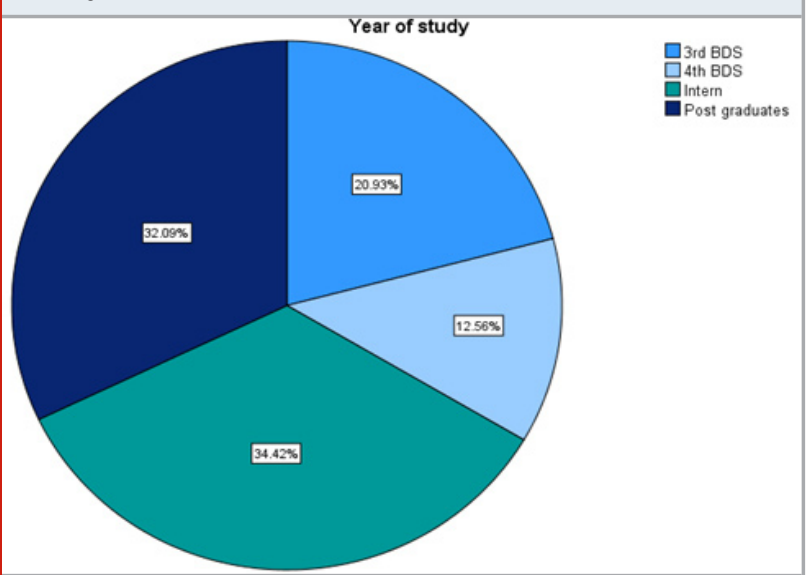


Figure 2 : Pie chart represents the responses received from the participants for the question, 'Which among the following are natural colloids?. About 54.42\% of the participants answered both albumin and plasma protein solutions, which is the correct answer.

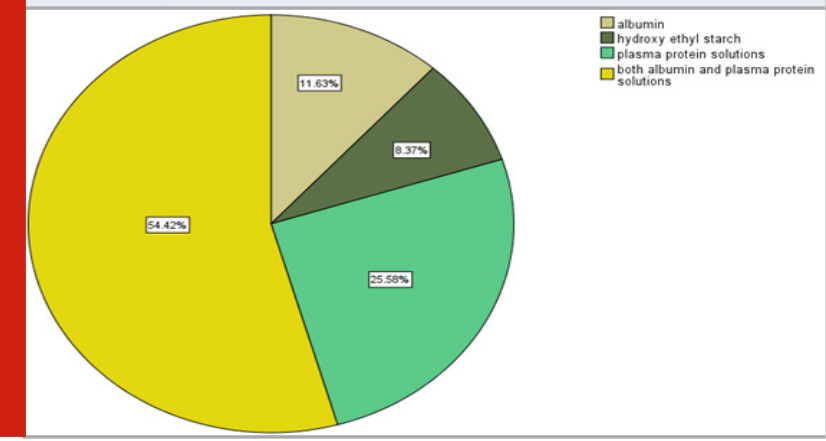

Figure 3: Bar chart representing the association between the year of study and the responses for the question, 'Which among the following are natural colloids?'. X axis represents the year of study and $\mathrm{Y}$ axis represents the number of responses. Chi square test was done and it was found to be statistically significant. Pearson Chi square value $=25.042 ; p$-value $=0.003(<0.05)$. Majority of postgraduates knew the correct answer which is both albumin and plasma protein solutions.

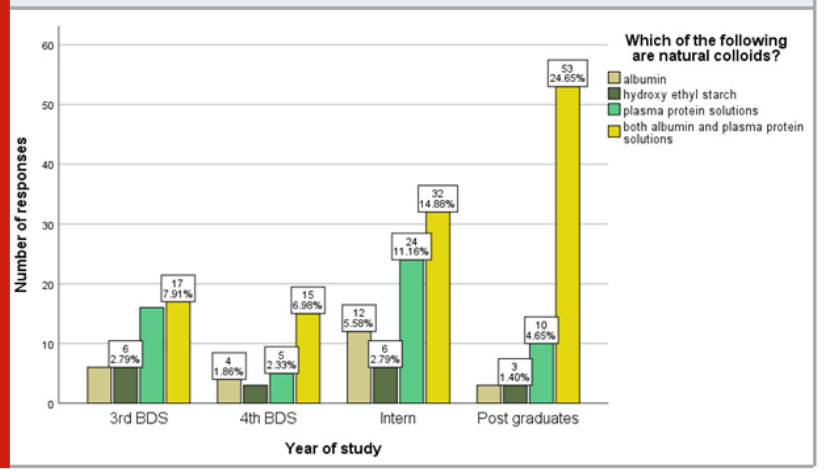

Figure 4: Pie chart represents the responses received from the participants for the question, 'Which among the following are synthetic colloids?'. About 54.88\% of the participants answered both dextran and hydroxyethyl starch, which is the correct answer.

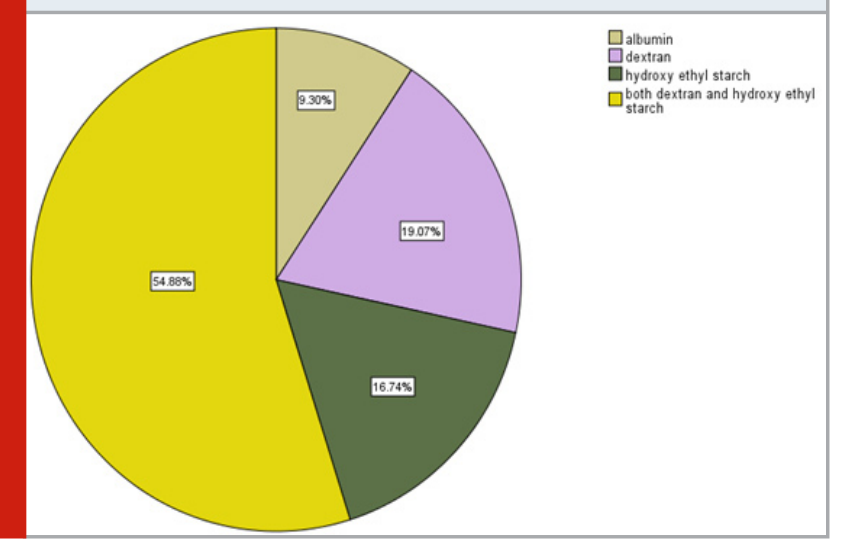

Figure 5 : Bar chart representing the association between the year of study and the responses for the question, 'Which among the following are synthetic colloids?' . X axis represents the year of study and $\mathrm{Y}$ axis represents the number of responses. Chi square test was done and it was found to be statistically significant. Pearson Chi square value $=42.337 ; \mathrm{p}$-value $=0.000(<0.05)$. Majority of postgraduates knew the correct answer which is both dextran and hydroxyethyl starch.

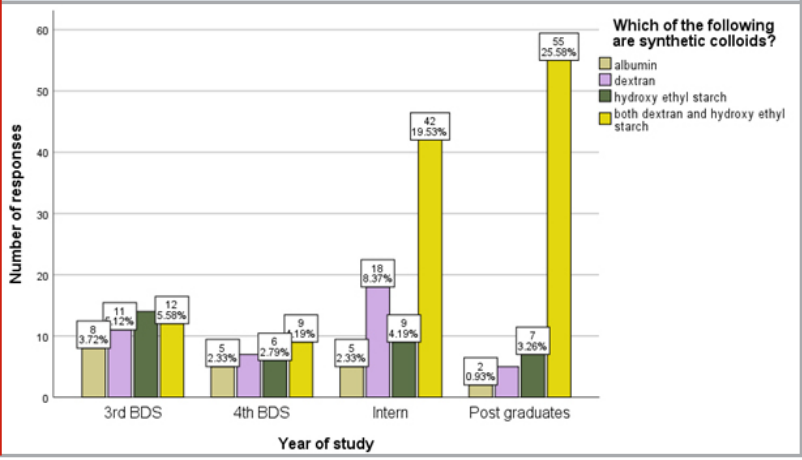

About $57.67 \%$ of the students were aware that synthetic colloids can be stored for long periods of time independent of storage temperature[Figure 6]. Out of the $57.67 \%, 26.05 \%$ were postgraduates, $18.6 \%$ were interns, 6.51\% were final year undergraduates and 6.51\% were third year undergraduate students. There was a significant association $(p=0.000)$ between the year of study and the response for the question, "Are you aware that synthetic colloids can be stored for long periods of time independent of storage temperature?' [Figure 7]. $70.7 \%$ were aware that synthetic colloids should be free of pyrogens and antigens [Figure 8]. Out of the 70.7\%, 26.98\% were interns, 25.58\% were postgraduates, 8.84\% were final year students and 9.3\% were third year undergraduate students.

Figure 6: Pie chart represents the responses received from the participants for the question, "Are you aware that synthetic colloids are capable of being stored for long periods of time independent of the storage temperature?'. About $57.67 \%$ of the students answered yes.

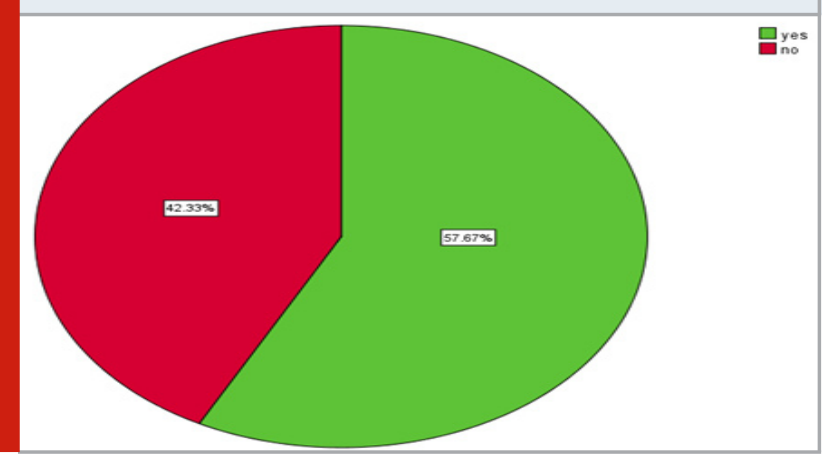

There was a significant association $(\mathrm{p}=0.000)$ between the year of study and the response for the question, 'Are you aware that synthetic colloids should be free of pyrogens and antigens?' [Figure 9]. Only $31.16 \%$ of the entire study 
population knew that Leuconostoc mesenteroides are the bacteria used in the production of dextrans [Figure 10]. Among them, $16.74 \%$ were postgraduates, 5.58\% were interns, $2.79 \%$ were final year undergraduates and $6.05 \%$ were third year undergraduate students. There was a significant association $(p=0.000)$ between the year of study and the response for the question, 'Which of the following bacteria are used in the production of dextrans?' [Figure 11].

Figure 7: Bar chart representing the association between the year of study and the responses for the question, 'Are you aware that synthetic colloids are capable of being stored for long periods of time independent of the storage temperature?'. $\mathrm{X}$ axis represents the year of study and $Y$ axis represents the number of responses. Chi square test was done and it was found to be statistically significant. Pearson Chi square value $=29.370 ; \mathrm{p}$-value $=0.000(<0.05)$. Majority of postgraduates answered yes, followed by interns.

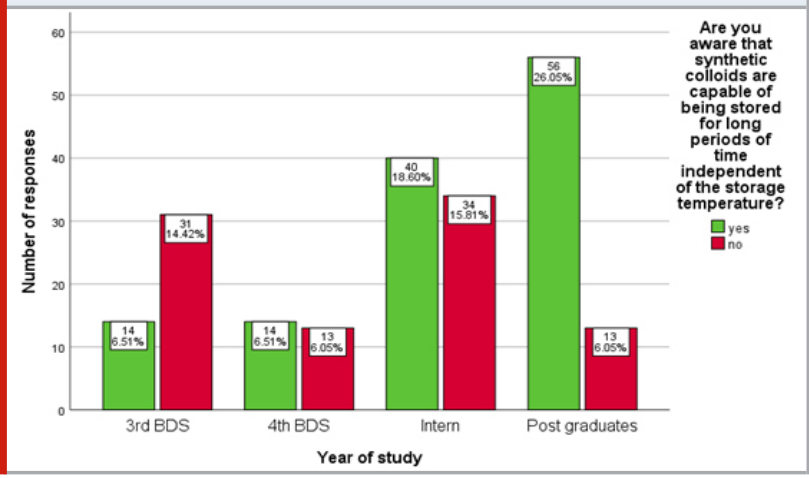

Figure 8: Pie chart represents the responses received from the participants for the question, "Are you aware that synthetic colloids should be free of pyrogens and antigens?'. About 70.7\% of the students answered yes.

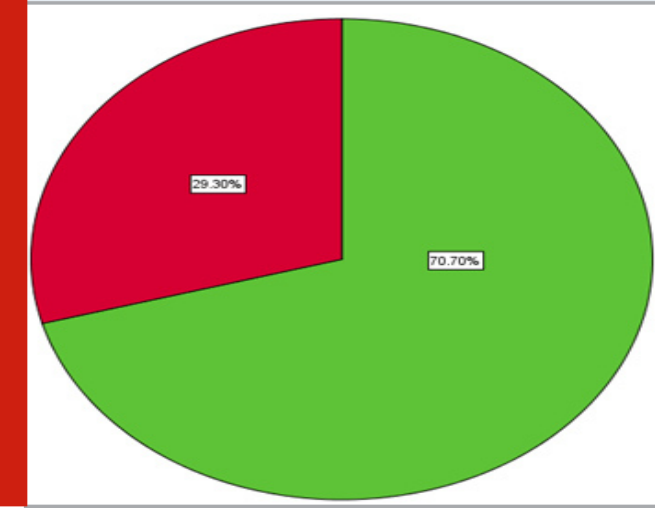

About 60\% were aware that gelatins are produced by the hydrolysis of animal collagens [Figure 12]. Among them, 24.19\% were postgraduates, 20.93\% were interns, $5.58 \%$ were final year undergraduates and 9.3\% were third year students. There was a significant association $(p=0.003)$ between the year of study and the response for the question, 'Are you aware that gelatins are produced by the hydrolysis of animal collagens?' [Figure 13].
Figure 9: Bar chart representing the association between the year of study and the responses for the question, 'Are you aware that synthetic colloids should be free of pyrogens and antigens?'. $X$ axis represents the year of study and $Y$ axis represents the number of responses. Chi square test was done and it was found to be statistically significant. Pearson Chi square value $=19.786$; $\mathrm{p}$-value $=$ $0.000(<0.05)$. Majority of interns answered yes, followed by postgraduates.

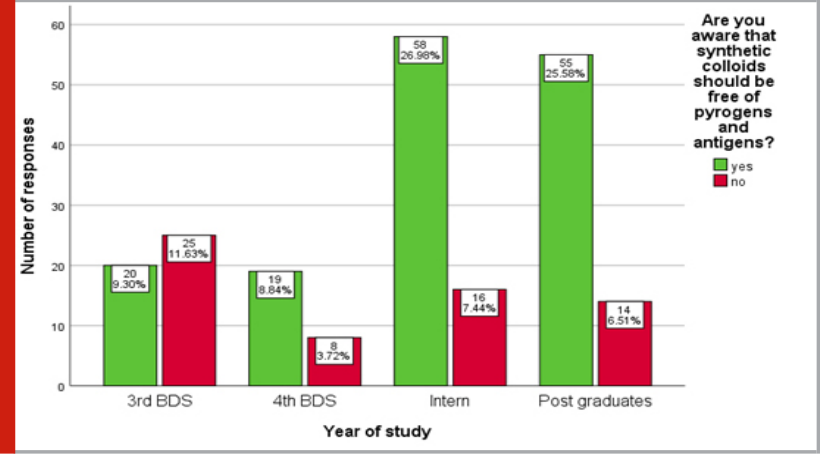

Figure 10: Pie chart represents the responses received from the participants for the question, 'Which of the following bacteria are used in the production of dextrans?'. About 31.16\% of the participants answered Leuconostoc mesenteroides, which is the correct answer.

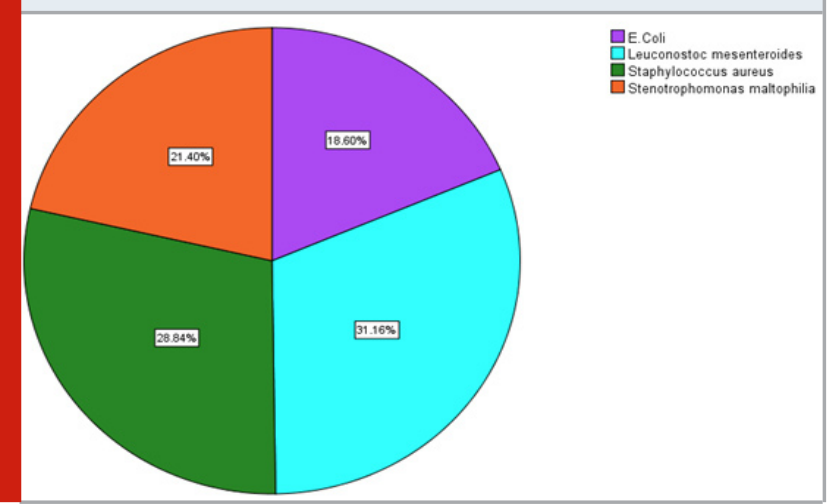

$70.23 \%$ were aware that the principal use of colloid plasma substitutes is in the treatment of hypovolaemia [Figure 14]. Out of the $70.23 \%, 27.44 \%$ were postgraduates, 24.19\% were interns, 6.51\% were final years and $12.09 \%$ were third year students. There was a significant association $(p=0.002)$ between the year of study and the response for the question, 'Are you aware that the principal use of colloid plasma substitutes is in the treatment of hypovolaemia?' [Figure 15]. Only 31.63\% of the dental students knew that the molecular weight of plasma protein is 50,000 Da [Figure 16].

Among them, 11.63\% were postgraduates, $11.63 \%$ were interns, 4.19\% were final year students and $4.19 \%$ were third year undergraduate students. There was no significant association $(p=0.472)$ between the year of study and the response for the question, 'What is the mean molecular weight of plasma protein?' [Figure 17]. About $35.81 \%$ of students were aware that the half life of 
Dextran 60 is six hours [Figure 18]. Among them, 16.74\% were postgraduates, $9.76 \%$ were interns, $4.19 \%$ were final year students and 5.12\% were third year students. There was no significant association $(p=0.561)$ between the year of study and the response for the question, 'What is the half life of Dextran 60?' [Figure 19]. Only 32.56\% of students knew that blood volume was increased the least by gelatin [Figure 20]. Among them, 13.02\% were postgraduates, $12.56 \%$ were interns, 3.26\% were final year students and $3.72 \%$ were third year students. There was no significant association $(p=0.447)$ between the year of study and the response for the question, 'Which among the following increases blood volume the least?' [Figure 21].

Figure 11: Bar chart representing the association between the year of study and the responses for the question, 'Which of the following bacteria are used in the production of dextrans?'. $\mathrm{X}$ axis represents the year of study and Y axis represents the number of responses. Chi square test was done and it was found to be statistically significant. Pearson Chi square value $=34.016 ; p$-value $=0.000(<0.05)$. Majority of postgraduates knew the correct answer which is Leuconostoc mesenteroides.

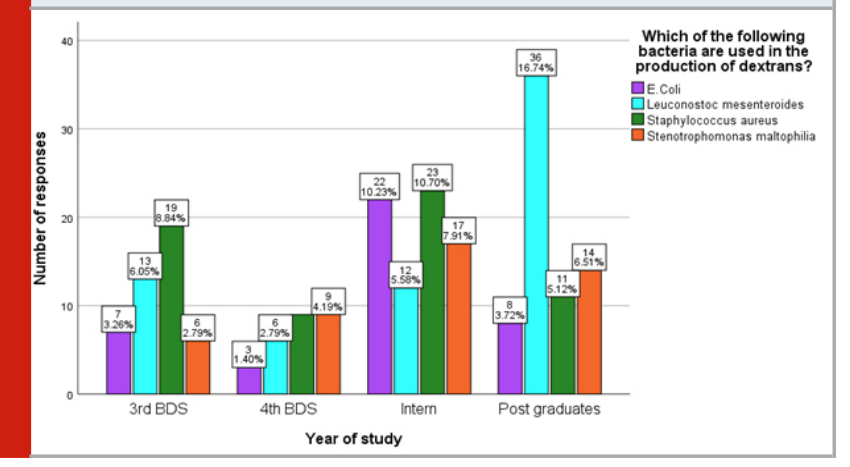

Figure 12: Pie chart represents the responses received from the participants for the question, 'Are you aware that gelatins are produced by the hydrolysis of animal collagen?'. About 60\% of the students answered yes.

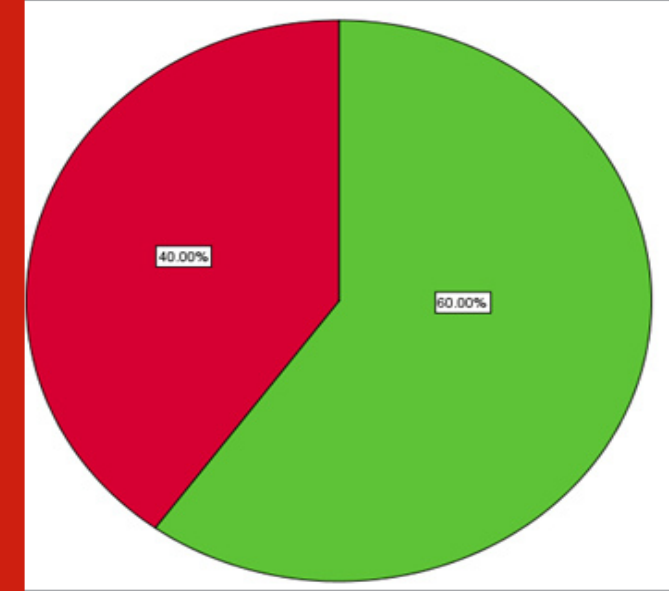

Figure 13: Bar chart representing the association between the year of study and the responses for the question, 'Are you aware that gelatins are produced by the hydrolysis of animal collagen?'. X axis represents the year of study and $\mathrm{Y}$ axis represents the number of responses. Chi square test was done and it was found to be statistically significant. Pearson Chi square value $=14.065$; $\mathrm{p}$-value $=0.003(<0.05)$. Majority of postgraduates answered yes, followed by interns.

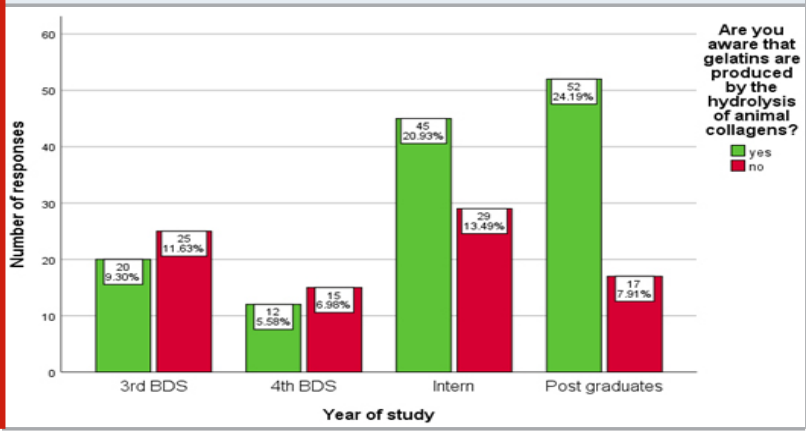

Figure 14: Pie chart represents the responses received from the participants for the question, "Are you aware that the principal use of colloid plasma substitutes is in the treatment of hypovolemia?'. About $70.23 \%$ of the students answered yes.

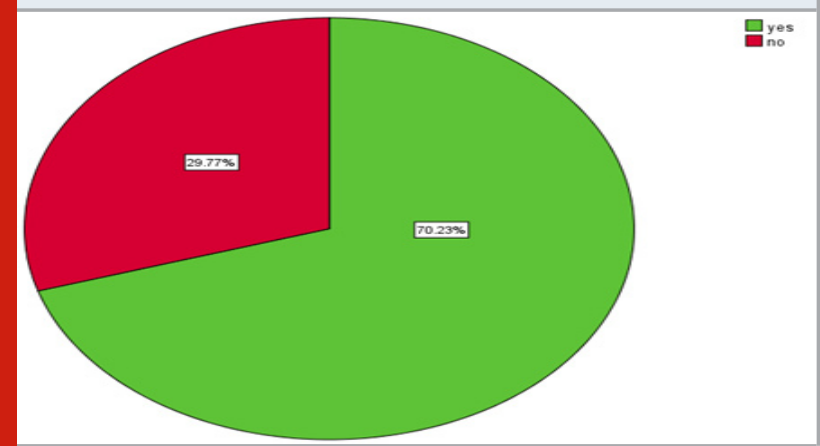

Figure 15: Bar chart representing the association between the year of study and the responses for the question, 'Are you aware that the principal use of colloid plasma substitutes is in the treatment of hypovolemia?'. $X$ axis represents the year of study and $Y$ axis represents the number of responses. Chi square test was done and it was found to be statistically significant. Pearson Chi square value $=15.403 ; p$-value $=0.002(<0.05)$. Majority of postgraduates answered yes, followed by interns.

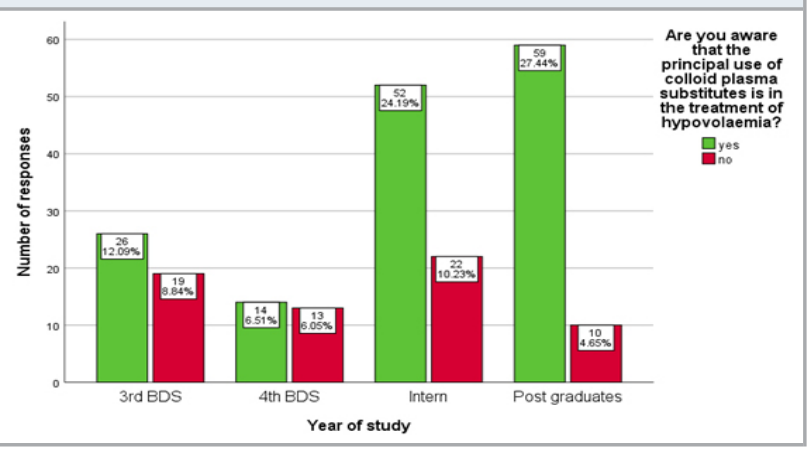


Figure 16: Pie chart represents the responses received from the participants for the question, 'What is the mean molecular weight of plasma protein?'. About 31.63\% of the participants answered $50,000 \mathrm{Da}$, which is the correct answer.

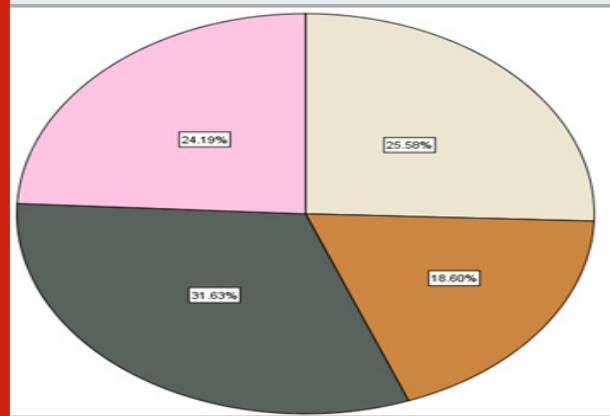

Figure 17 : Bar chart representing the association between the year of study and the responses for the question, 'What is the mean molecular weight of plasma protein?'. $\mathrm{X}$ axis represents the year of study and $\mathrm{Y}$ axis represents the number of responses. Chi square test was done and it was found to be statistically not significant. Pearson Chi square value $=8.630 ; p$-value $=0.472(>0.05)$. Majority of postgraduates knew the correct answer which is 50,000 Da.

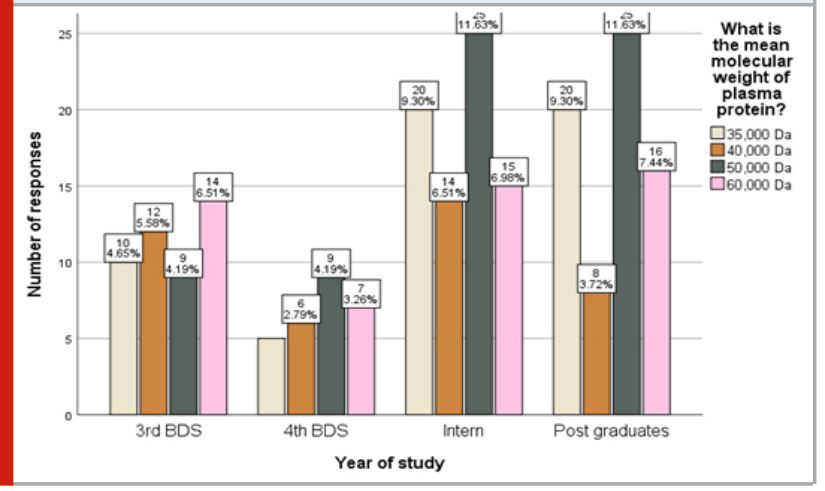

Figure 18: Pie chart represents the responses received from the participants for the question, 'What is the half life of Dextran 60?'. About 35.81\% of the participants answered 6 hours, which is the correct answer.

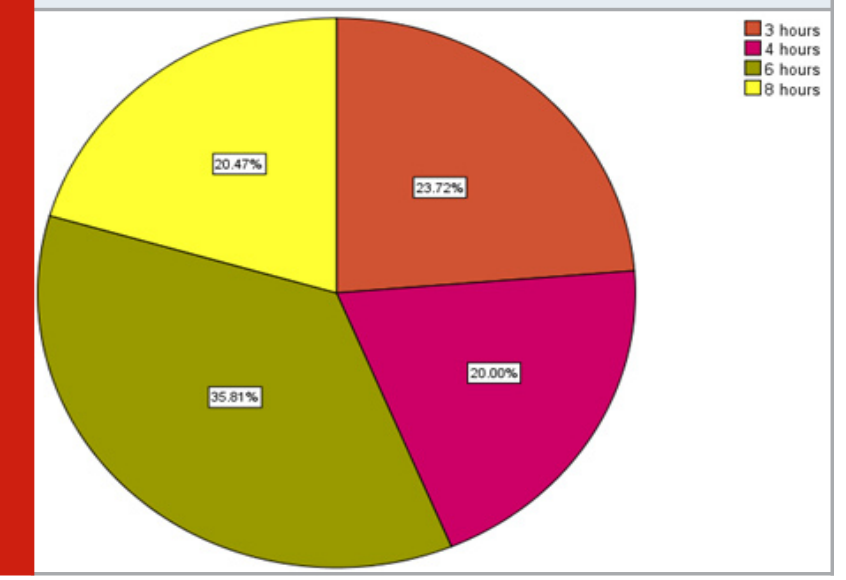

Figure 19 : Bar chart representing the association between the year of study and the responses for the question, 'What is the half life of Dextran 60?'. X axis represents the year of study and $\mathrm{Y}$ axis represents the number of responses. Chi square test was done and it was found to be statistically not significant. Pearson Chi square value $=$ $16.584 ; p$-value $=0.561(>0.05)$. Majority of postgraduates knew the correct answer which is 6 hours.

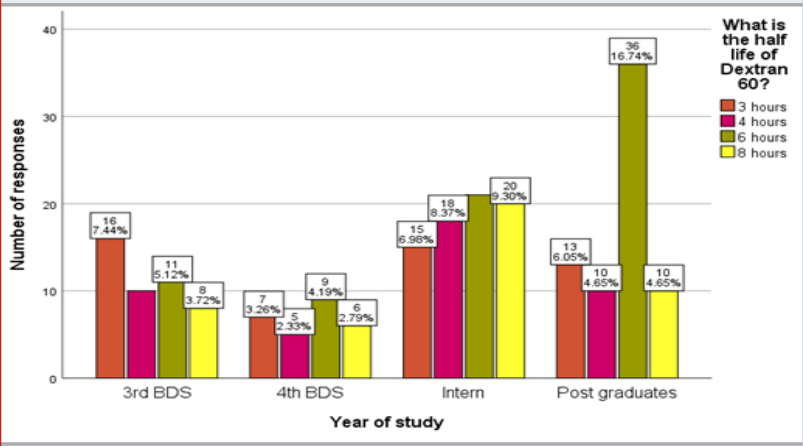

Figure 20: Pie chart represents the responses received from the participants for the question, 'Which among the following increases blood volume the least?'. About $32.56 \%$ of the participants answered gelatin, which is the correct answer.

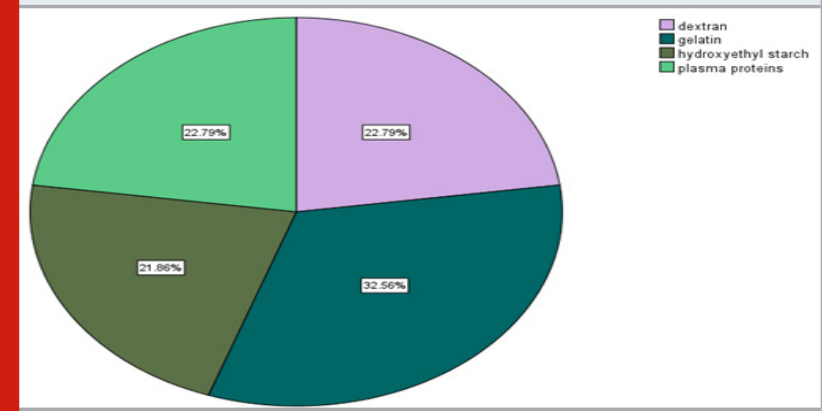

Figure 21: Bar chart representing the association between the year of study and the responses for the question, 'Which among the following increases blood volume the least?'. X axis represents the year of study and Y axis represents the number of responses. Chi square test was done and it was found to be statistically not significant. Pearson Chi square value $=8.894 ;$-value $=0.447(>0.05)$. Majority of postgraduates knew the correct answer which is gelatin.

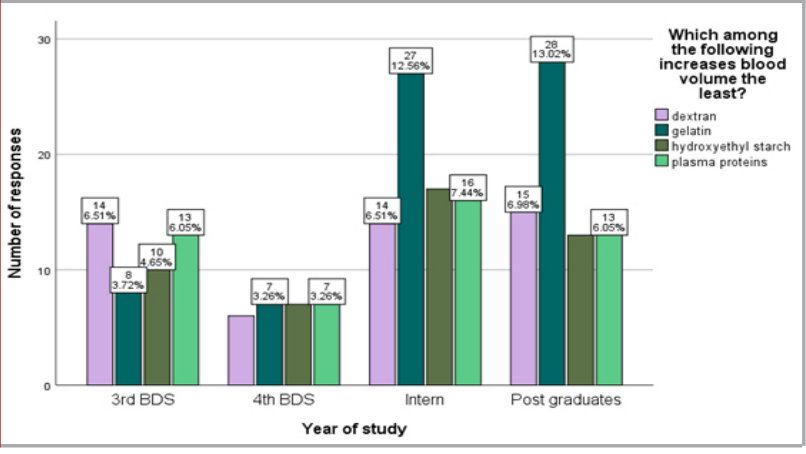


Figure 22: Pie chart represents the responses received from the participants for the question, 'Are you aware that anaphylactoid reactions may occur after administration of plasma substitutes?'. About 74.8\% of the students answered yes.

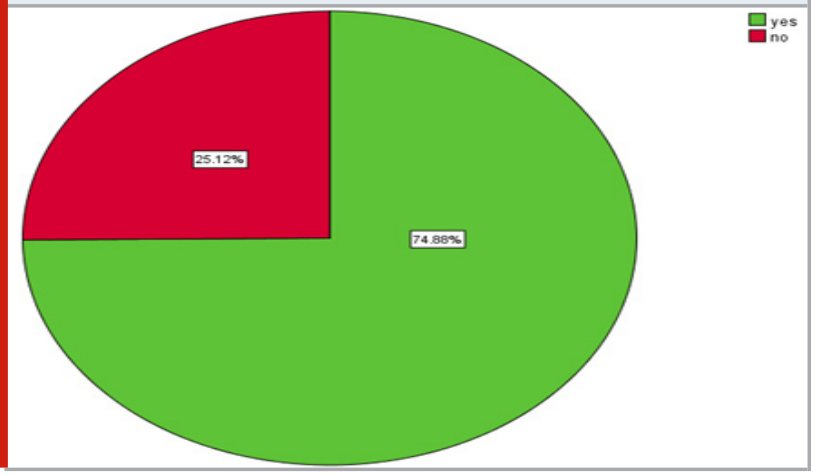

Figure 23: Bar chart representing the association between the year of study and the responses for the question, 'Are you aware that anaphylactoid reactions may occur after administration of plasma substitutes?'. $\mathrm{X}$ axis represents the year of study and $Y$ axis represents the number of responses. Chi square test was done and it was found to be statistically significant. Pearson Chi square value = $21.146 ; p$-value $=0.000(<0.05)$. Majority of postgraduates answered yes, followed by interns.

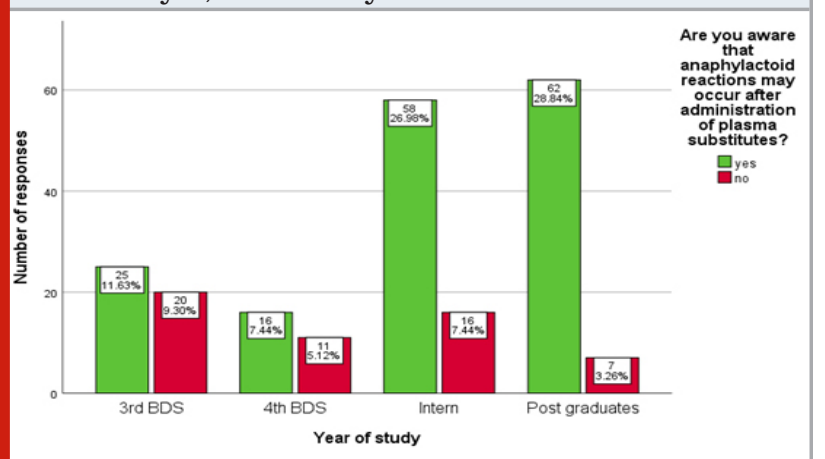

Figure 24: Pie chart represents the responses received from the participants for the question, 'Are you aware that histamine is released in humans by gelatin than dextran?'. Only $28.37 \%$ of the students answered yes.

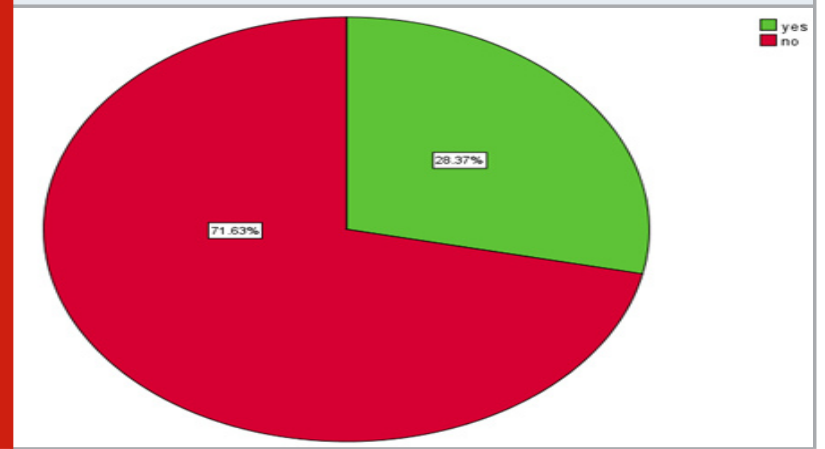

About $74.88 \%$ of dental students were aware that anaphylactoid reactions may occur after administration

of plasma substitutes [Figure 22]. Out of 74.88\%, 28.84\% were postgraduates, 26.98\% were interns, 7.44\% were final year students and 11.63\% were third year students. There was a significant association $(p=0.000)$ between the year of study and the response for the question, 'Are you aware that anaphylactoid reactions may occur after administration of plasma substitutes?' [Figure 23]. Only $28.37 \%$ of students were aware that histamine is released in humans following administration of gelatin than dextran [Figure 24]. Among them, 13.02\% were postgraduate students, $8.37 \%$ were interns, $3.26 \%$ were final year students and 3.72\% were third year students. There was no significant association $(p=0.421)$ between the year of study and the response for the question, 'Are you aware that histamine is released in humans following administration of gelatin than dextran?' [Figure 25]. About 46.05\% of students were aware that anaphylactoid reactions to dextrans are related to antidextran antibodies such as IgG and IgM [Figure 26]. Out of the $46.05 \%$, $18.6 \%$ were postgraduates, $12.56 \%$ were interns, 6.51\% were final year students and $8.37 \%$ were third year students.

Figure 25: Bar chart representing the association between the year of study and the responses for the question, 'Are you aware that histamine is released in humans by gelatin than dextran?'. $X$ axis represents the year of study and $\mathrm{Y}$ axis represents the number of responses. Chi square test was done and it was found to be statistically not significant. Pearson Chi square value $=8.221 ; \mathrm{p}$-value $=$ $0.421(>0.05)$. Majority of postgraduates answered yes, followed by interns.

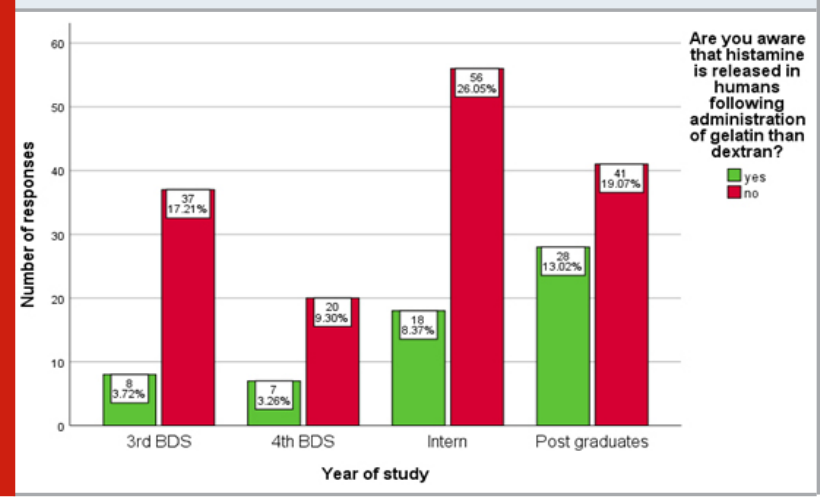

Figure 26: Pie chart represents the responses received from the participants for the question, 'Are you aware that anaphylactoid reactions to dextran are related to anti dextran antibodies such as IgG and IgM?'. About 46.05\% of the students answered yes.

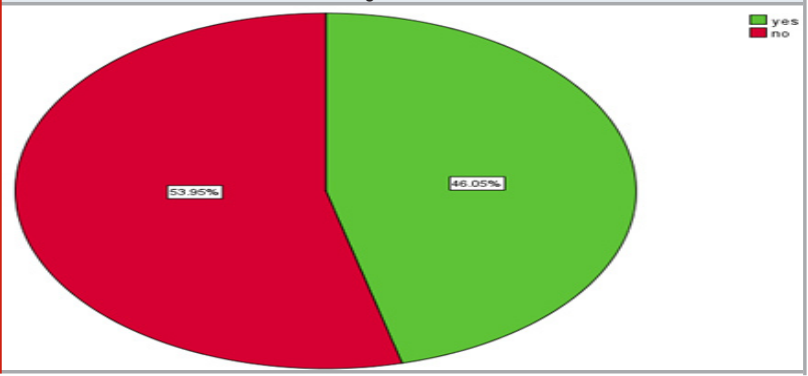


Figure 27: Bar chart representing the association between the year of study and the responses for the question, 'Are you aware that anaphylactoid reactions to dextran are related to anti dextran antibodies such as IgG and IgM?'. $\mathrm{X}$ axis represents the year of study and $\mathrm{Y}$ axis represents the number of responses. Chi square test was done and it was found to be statistically not significant. Pearson Chi square value $=7.700 ;$ p-value $=0.531(>0.05)$. Majority of postgraduates answered yes, followed by interns.

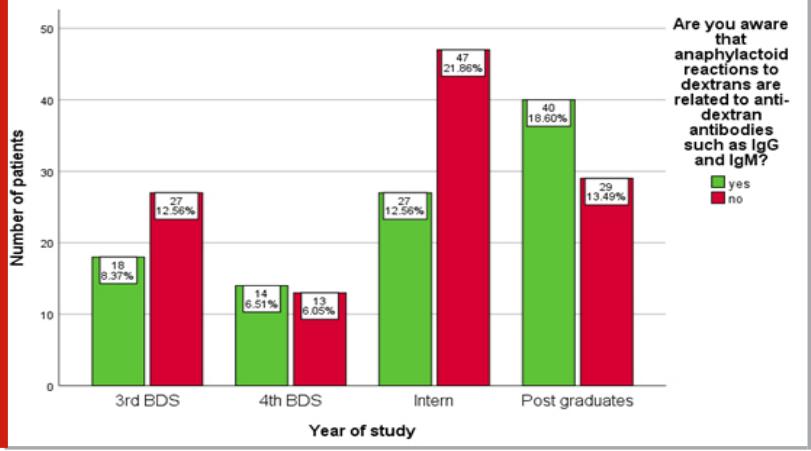

Figure 28: Pie chart represents the responses received from the participants for the question, 'What is the mechanism of action involved in the anaphylactoid reaction to hydroxyethyl starch?'. About $32.56 \%$ of the participants answered complement activation, which is the correct answer.

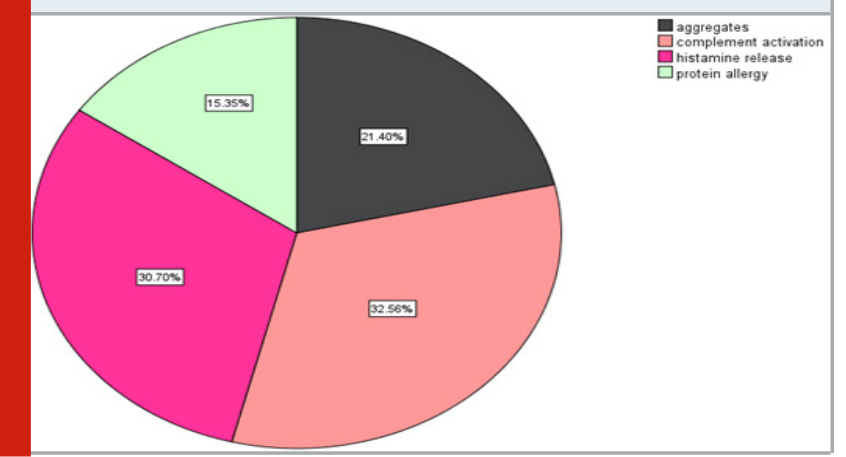

There was no significant association $(p=0.531)$ between the year of study and the response for the question, 'Are you aware that anaphylactoid reactions to dextrans are related to anti-dextran antibodies such as IgG and IgM?' [Figure 27]. Only 32.56\% of students knew that complement activation is the mechanism involved in the anaphylactoid reaction to hydroxyethyl starch [Figure 28]. Among them, 13.02\% were postgraduates, 11.63\% were interns, 4.19\% were final year students and 3.72\% were third year students. There was a significant association $(p=0.000)$ between the year of study and the response for the question, 'What is the mechanism involved in the anaphylactoid reaction to hydroxyethyl starch?' [Figure 29]. About 43.72\% of students were aware that human albumin should be used as an alternate solution in case of severe anaphylaxis to plasma substitutes [Figure 30]. Out of the $43.72 \%, 16.74 \%$ were postgraduates, $13.96 \%$ were interns, $4.65 \%$ were final year students and $8.37 \%$ were third year undergraduate students. There was no significant association $(p=0.579)$ between the year of study and the response for the question, 'In case of severe anaphylaxis to plasma substitutes, which solution should be used as an alternative?' [Figure 31]

This study was done to evaluate the level of awareness of plasma substitutes among dental students, including both undergraduates and postgraduates. Considering the responses, it can be interpreted that the knowledge regarding plasma substitutes is above average among dental students. Also, postgraduate students were more knowledgeable than undergraduate students. Interns showed more awareness than third year or final year undergraduate students. Students were also more aware of the anaphylactoid reactions associated with the administration of plasma substitutes.

Figure 29 : Bar chart representing the association between the year of study and the responses for the question, 'What is the mechanism of action involved in the anaphylactoid reaction to hydroxyethyl starch?'. X axis represents the year of study and $\mathrm{Y}$ axis represents the number of responses. Chi square test was done and it was found to be statistically significant. Pearson Chi square value $=21.539$; $\mathrm{p}$-value $=0.000(<0.05)$. Majority of postgraduates knew the correct answer which is complement activation.

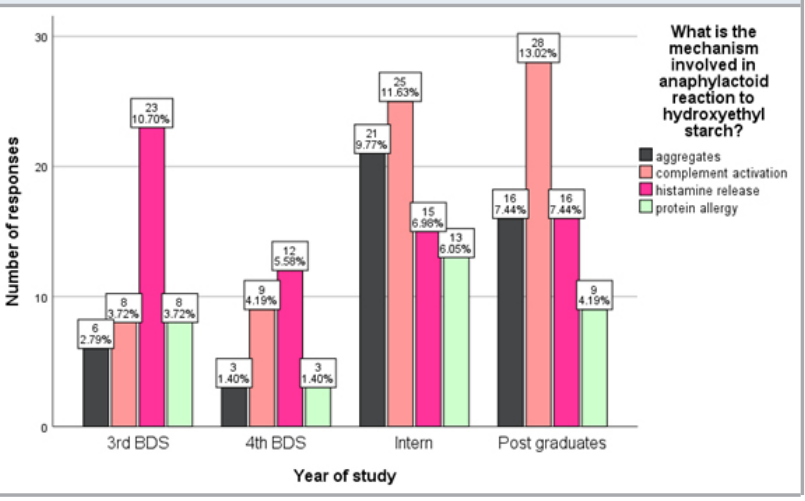

Figure 30: Pie chart represents the responses received from the participants for the question, 'In case of severe anaphylaxis to plasma substitutes, which solution should be used as an alternative?'. About $43.72 \%$ of the participants answered human albumin, which is the correct answer.

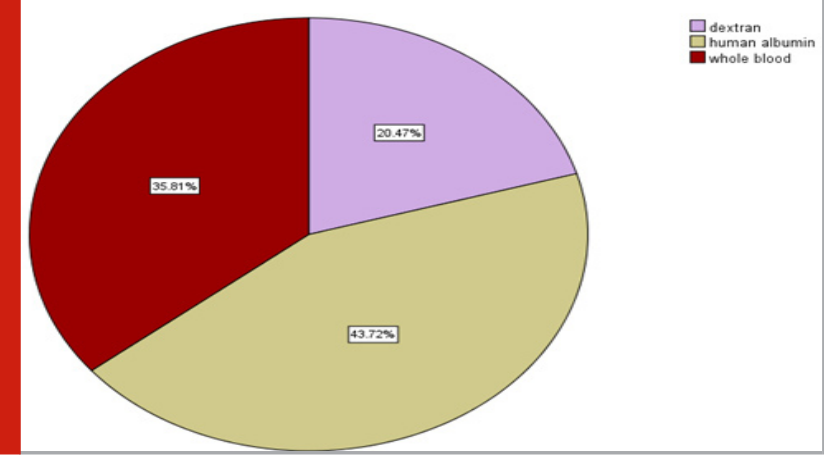


Figure 31: Bar chart representing the association between the year of study and the responses for the question, 'In case of severe anaphylaxis to plasma substitutes, which solution should be used as an alternative?'. $\mathrm{X}$ axis represents the year of study and $\mathrm{Y}$ axis represents the number of responses. Chi square test was done and it was found to be statistically not significant. Pearson Chi square value $=32.098 ; \mathrm{p}$-value $=0.579(>0.05)$. Majority of postgraduates knew the correct answer which is human albumin.

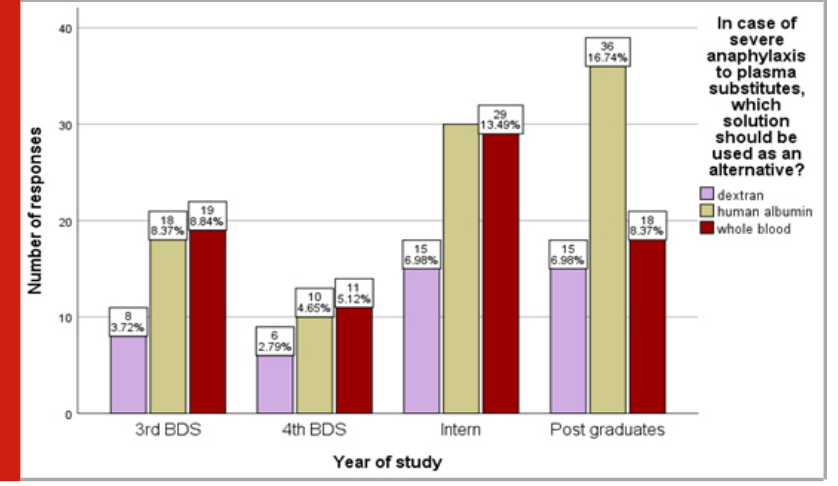

The plasma substitutes of greatest clinical use are: the natural colloids (plasma protein solutions, human albumin) and the synthetic colloids (dextrans, gelatins and hydroxyethyl starches) prepared from materials derived from animals or plants. In our study about $54.42 \%$ and 54.88\% knew the various natural and synthetic colloids. Any synthetic colloid should have the following desirable properties. First, it should maintain an adequate colloid osmotic pressure with a half-life of several hours. Second, it should be stable and capable of being stored for long periods independent of the storage temperature. Third, it should be free of pyrogens and antigens. Finally, its metabolism and elimination should not affect the organism and it should not cause haemolysis or red cell agglutination(Moe, 1970). Knowledge regarding these properties among dental students was average.

Very few students knew about the production of dextrans and gelatins. They are produced by the bacterium Leuconostoc mesenteroides B512 from an agar-sugar compound to which a yeast extract is added as a source of nitrogen(Moffitt, 1975). Gelatins are prepared by the hydrolysis of animal collagens. There are three modified solutions available: a modified fluid gelatin, a urealinked gelatin and an oxypoly gelatin. Starch solutions prepared by the acid hydrolysis of corn or soya may be used as plasma expanders only after the introduction of hydroxyethyl groups into the glucose units. To obtain a satisfactory half-life in the circulation the commercially available solutions have hydroxyethyl units attached to 70\% of the glucose units(Ring et al., 1976).

Majority of the students were aware that the principal indication for the use of colloid plasma substitutes is in the treatment of hypovolaemia. Only few of them knew about the half life of plasma substitutes. The duration of action of Dextran 60 is about $6 \mathrm{~h}$ as is that of hydroxyethyl starch (HES), whilst gelatins and Dextran 40 last only 2-3 h(Hankeln et al., 1997). The increase in blood volume is least with gelatin(Ring and Messmer, 1977) and only about $32.56 \%$ of dental students were aware of this.

There was increased awareness among students regarding the anaphylactoid reactions associated with plasma substitutes. A prospective comparative study in 31 hospitals in Southern Bavaria observed 69 anaphylactoid reactions following 100906 units of the commonly used colloidal plasma substitutes. There were differences between the agents: following plasma proteins there were reactions in $0.014 \%$ of patients, following dextran in $0.032 \%$, following gelatins in $0.115 \%$ and following hydroxyethyl starch in $0.085 \%$. The types of anaphylactoid reaction seen varied in intensity from skin reactions with flushing and mild urticaria to more severe effects such as shock and cardiac and respiratory arrest. The frequencies of life-endangering reactions were: $0.003 \%$ for plasma proteins, $0.008 \%$ for dextran, $0.038 \%$ for gelatins and $0.001 \%$ for hydroxyethyl starch(Johnson and Laurell, 1974).

The extent to which histamine release is involved in these reactions was investigated and it was concluded that histamine can be released in humans by gelatin and to a smaller degree by dextran(Lorenz et al., 1973). However, knowledge regarding the role of histamine was very poor among the students as only $28.37 \%$ were aware of this. There was also very little awareness among the students regarding the mechanism involved in anaphylaxis of various plasma substitutes(Lorenz et al., 1976). If there is only mild urticaria, stopping the infusion usually is all that is needed, but if the urticaria is severe, calcium antihistamines and corticosteroids should be used. High doses of corticosteroids should also be given and the plasma volume should be expanded using a human albumin solution. Considering cases of severe anaphylaxis, only about $43.72 \%$ of students were aware that human albumin has to be used as a plasma expander(Lorenz et al., 1972).

According to this study, post graduates had more awareness followed by interns. Limitations of this study include dishonest answers in the questionnaire by respondents and usage of a single online survey platform in a single university setting. Future studies should aim at conducting surveys using multiple online survey platforms to include more participants in different university settings.

\section{CONCLUSION}

This study shows that postgraduates have the most awareness of plasma substitutes among all dental students. Educational workshops, conferences and CDE's are essential for both undergraduates and postgraduates to improve their knowledge regarding various plasma substitutes and their adverse reactions. 


\section{ACKNOWLEDGEMENTS}

The authors sincerely acknowledge Saveetha Dental College and Hospitals, Saveetha Institute of Medical and Technical Sciences for granting permission to conduct this questionnaire based study among dental students.

Author Contributions: All authors contributed equally to the design and implementation of the research, analysis of the results and to the writing of the manuscript.

Conflict of Interest: The authors declare that they have no conflict of interest.

\section{REFERENCES}

Anbu, R. T. et al. (2019) 'Comparison of the Efficacy of Three Different Bone Regeneration Materials: An Animal Study', European journal of dentistry, 13(1), pp. 22-28.

Ariga, P. et al. (2018) 'Determination of Correlation of Width of Maxillary Anterior Teeth using Extraoral and Intraoral Factors in Indian Population: A Systematic Review', World Journal of Dentistry, 9(1), pp. 68-75.

Ashok, V. and Ganapathy, D. (2019) 'A geometrical method to classify face forms', Journal of oral biology and craniofacial research, 9(3), pp. 232-235.

Chattri, G. L. (2012) 'Plasma Volume Expanders', Pediatric Drug Doses, pp. 175-175. doi: 10.5005/jp/ books/11551_39.

Duraisamy, R. et al. (2019) 'Compatibility of Nonoriginal Abutments With Implants: Evaluation of Microgap at the Implant-Abutment Interface, With Original and Nonoriginal Abutments', Implant dentistry, 28(3), pp. 289-295.

Evaluation of Corrosive Behavior of Four Nickelchromium Alloys in Artificial Saliva by Cyclic Polarization Test:An in vitro Study' (2017) World Journal of Dentistry, 8(6), pp. 477-482.

Ganapathy, D. M., Kannan, A. and Venugopalan, S. (2017) 'Effect of Coated Surfaces influencing Screw Loosening in Implants: A Systematic Review and Meta-analysis', World Journal of Dentistry, 8(6), pp. 496-502.

Grobbelaar, B. G. and Smart, E. (1967) 'The Incidence of Isosensitization Following Blood Transfusion', Transfusion, pp. 152-156. doi: 10.1111/j.15372995.1967.tb04857.x.

Gruber, U. F. (1969) 'Volume Replacement Using Blood', Blood Replacement, pp. 19-40. doi: 10.1007/978-3-64249790-2_6.

Gupta, P., Ariga, P. and Deogade, S. C. (2018) 'Effect of Monopoly-coating Agent on the Surface Roughness of a Tissue Conditioner Subjected to Cleansing and Disinfection: A Contact Profilometric Study', Contemporary clinical dentistry, 9(Suppl 1), pp. S122-
S126.

Hankeln, K. et al. (1997) '[A comparative study of the effectiveness of 5\% human albumin and 10\% hydroxyethyl starch (HAES-steril) for correcting hemodynamics and 02 transport in surgical interventions]', Anesteziologiia i reanimatologiia, (1), pp. 23-26.

Jain, A. R. (2017a) 'Clinical and Functional Outcomes of Implant Prostheses in Fibula Free Flaps', World Journal of Dentistry, 8(3), pp. 171-176.

Jain, A. R. (2017b) 'Prevalence of Partial Edentulousness and Treatment needs in Rural Population of South India', World Journal of Dentistry, 8(3), pp. 213-217.

Johnson, U. and Laurell, A. B. (1974) 'Activation of complement in anaphylactoid reactions in connection with infusion of dextran', Scandinavian journal of immunology, 3(5), pp. 673-676.

Kirch, W., Kühler, H. and Gizycki, C. (1979) 'The Influence of Colloidal Plasma Substitutes on Spontaneous and Induced Platelet Aggregation', Acute Care, pp. 156-160. doi: 10.1007/978-3-642-67211-8_25.

Lamke, L.-0. and Liljedahl, S.-O. (1976) 'Plasma volume changes after infusion of various plasma expanders', Resuscitation, pp. 93-101. doi: 10.1016/03009572(76)90029-0.

Lorenz, W. et al. (1972) 'A Sensitive and Specific Method for the Determination of Histamine in Human Whole Blood and Plasma', Hoppe-Seyler's Zeitschrift für physiologische Chemie, pp. 911-920. doi: 10.1515/ bchm2.1972.353.1.911.

Lorenz, W. et al. (1973) 'Plasma histamine determination in man and dog following the infusion of plasma substitutes: Models for histamine release under pathophysiological conditions', Agents and Actions, pp. 183-184. doi: 10.1007/bf01965740.

Lorenz, W. et al. (1976) 'HISTAMINE RELEASE IN HUMAN SUBJECTS BY MODIFIED GELATIN (HAEMACCEL) AND DEXTRAN: AN EXPLANATION FOR ANAPHYLACTOID REACTIONS OBSERVED UNDER CLINICAL CONDITIONS?', British Journal of Anaesthesia, pp. 151-165. doi: 10.1093/bja/48.2.151. Moe, P. J. (1970) 'BLOOD-VOLUME REPLACEMENT', Acta Anaesthesiologica Scandinavica, pp. 70-78. doi: 10.1111/j.1399-6576.1970.tb00881.x.

Moffitt, E. A. (1975) 'Blood substitutes', Canadian Anaesthetists' Society journal, 22(1), pp. 12-19.

Ranganathan, H., Ganapathy, D. M. and Jain, A. R. (2017) 'Cervical and Incisal Marginal Discrepancy in Ceramic Laminate Veneering Materials: A SEM Analysis', Contemporary clinical dentistry, 8(2), pp. 272-278.

Ring, J. et al. (1976) 'Anaphylactoid reactions due to hydroxyethyl starch infusion', European 
surgical research. Europaische chirurgische Forschung. Recherches chirurgicales europeennes, 8(5), pp. 389399.

Ring, J. and Messmer, K. (1977) 'Incidence and severity of anaphylactoid reactions to colloid volume substitutes', The Lancet, 1(8009), pp. 466-469.

Sayman, W. A. and Garrott Allen, J. (1959) 'Blood, Plasma and Expanders of Plasma Volume in the Treatment of Hemorrhagic Shock', Surgical Clinics of North America, pp. 133-143. doi: 10.1016/s00396109(16)35690-0.

Sewta, R. (2009) 'Plasma Volume Expanders', Fundamentals of Operative Surgery, pp. 276-276. doi: 10.5005/jp/books/10317_28.

Solanke, T. F., Khwaja, M. S. and Madojemu, E. I. (1971) 'Plasma volume studies with four different plasma volume expanders', Journal of Surgical Research, pp. 140-143. doi: 10.1016/0022-4804(71)90038-2.

Stacca, R. et al. (1989) 'Plasma expanders in hemorrhagic shock', Resuscitation, pp. 153-154. doi: 10.1016/03009572(89)90013-0.

Stehling, L. C., Zauder, H. L. and Rogers, W. (1975) 'Intraoperative Autotransfusion', Anesthesiology, pp. 337-345. doi: 10.1097/00000542-197509000-00011. Underwood, R. J., Gowing, D. and Johnston, G. I. (1967) 'Effect of changes in blood volume on body impedance', Anesthesiology, 28(2), pp. 426-431.

Varghese, S. S., Ramesh, A. and Veeraiyan, D. N. (2019) 'Blended Module-Based Teaching in Biostatistics and Research Methodology: A Retrospective Study with Postgraduate Dental Students', Journal of dental education, 83(4), pp. 445-450. 